\title{
Breastmilk Metabolomics: Bridging the Gap between Maternal Nutrition and Infant Health Outcomes
}

\author{
Rodolfo Sumayao, Jr.*, Sol Joaquin Benigno, Gerald Anthony Jaen, Marilen Martin, \\ Charmaine Sagayap \\ Chemistry Department, De La Salle University, 2401 Taft Avenue, Manila 0922
}

\begin{abstract}
Breastmilk (BM) is the primary source of nutrition for the newborn infant and its first six months of life. Although the importance of BM in the proper growth and development of infants has previously been recognized in various research studies, it was not until various metabolites were probed in BM that researchers discovered the various beneficial effects of BM beyond its nutritive value. Metabolomics has emerged as a discipline which aims to comprehensively profile various metabolites in food and biological fluids. Although still in its incipient stages, BM metabolomics has provided invaluable insights into the chemical interaction between mother and infant. NMR- and MS-based techniques have made it possible to explore the metabolome of $\mathrm{BM}$ and link it to various aspects of maternal phenotype and nutrition and breastfed infant health outcomes. In addition, recent developments in analytical approaches for BM metabolite analysis and metabolomic data analysis have allowed researchers to increase the coverage of detected metabolites using multiple platforms and have supported its functional characterization which aids in investigation of the clinical and biological importance of metabolites. These advancements can potentially aid in the development of strategies to promote healthy feeding practices for infants or novel therapeutic and nutrition advances in pediatric research.
\end{abstract}

Keywords: breastmilk; metabolomics; metabolites

Abbreviations: BM, breastmilk; $M S$, mass spectrometry; NMR, nuclear magnetic resonance; HMO, human milk oligosaccharides; PCA, principal component analysis

\section{INTRODUCTION}

Breastmilk (BM) remains to be an unequalled food that meets the nutritional requirements of a newborn infant (Sumayao et al., 2009; WHO, 2003). It contains the optimal balance of nutrients and nonnutrient factors to support growth, development, and immunity of the infant. The importance and benefits of breastmilk are emphasized by the World Health Organization (WHO) which advocates initiation of breastfeeding within the first hour after the birth, exclusive breastfeeding for the first six months, and continued breastfeeding for two years or more (WHO, 2003). While the benefits of BM and the exclusive or frequent breastfeeding in infants were demonstrated in various clinical and epidemiological studies (Horta \& Victora, 2013; Allen \& Hector 2005), these studies did not provide strong correlation or causation between various biochemical components in BM and their beneficial effects in infants beyond promotion of optimal nutrition. This presents a gap between 
lactating mothers and breastfed infants in terms of their biochemical interaction and the mechanistic link between the various chemical factors in BM and infant health outcomes.

The biochemical composition of $\mathrm{BM}$ is complex which includes normal nutrients (i.e. carbohydrates, proteins, lipids, vitamins, and minerals) and various biologically active compounds (i.e. hormones, cytokines, antimicrobial peptides, antibodies, etc.) (Villaseñor et al., 2014). Furthermore, the composition of breastmilk is highly variable as it changes from woman to woman and within an individual mother constantly during lactation (Marincola et al., 2015). These dynamic changes in $\mathrm{BM}$ composition are essential to satisfy the nutritional and physiological requirements of a growing infant. Furthermore, a growing body of evidence suggests that nutrition during early development is not only critical for the general health of an infant in the shortterm as it may also influence long-term effects on health, disease predisposition, and mortality risks in adulthood, also known as 'metabolic programming' (Marincola et al., 2015). These underscore the importance of determining the $\mathrm{BM}$ composition since it may provide insights into the short- and long-term health outcomes of an infant.

Given the complexity of BM composition and the importance of BM in infant's health and proper development, researchers have placed great interests in unraveling the metabolome of BM. The term 'metabolomics' refers to the screening of lowmolecular weight molecules (i.e. metabolites) in complex biological fluids in an attempt to provide a 'snapshot' of the metabolism or a 'metabolic fingerprint', which can reflect the physiological state of the organism under study or reveal information on disease phenotypes. One of the strengths of comprehensive metabolite profiling is that it can reflect the downstream activity of genes (genome) or proteins (proteome), hence it can reveal more functional information than just a mere 'snapshot' of metabolism (Brennan, 2016) (Figure 1). The organism's metabolome reflects both genetic and exogenous factors, such as drugs, toxins, gut microbiota activity, and, most predominantly, diet (Astarita and Langridge, 2013). Therefore, the inventory of metabolites in a biological system can offer a high level of functional description that transcends pure genomics, transcriptomics, or proteomics information.

There are basically two major approaches in metabolomics analyses. These approaches are the discovery metabolomics (also known as untargeted metabolomics) and targeted metabolomics (Xia \& Wishart, 2011). Discovery metabolomics aims

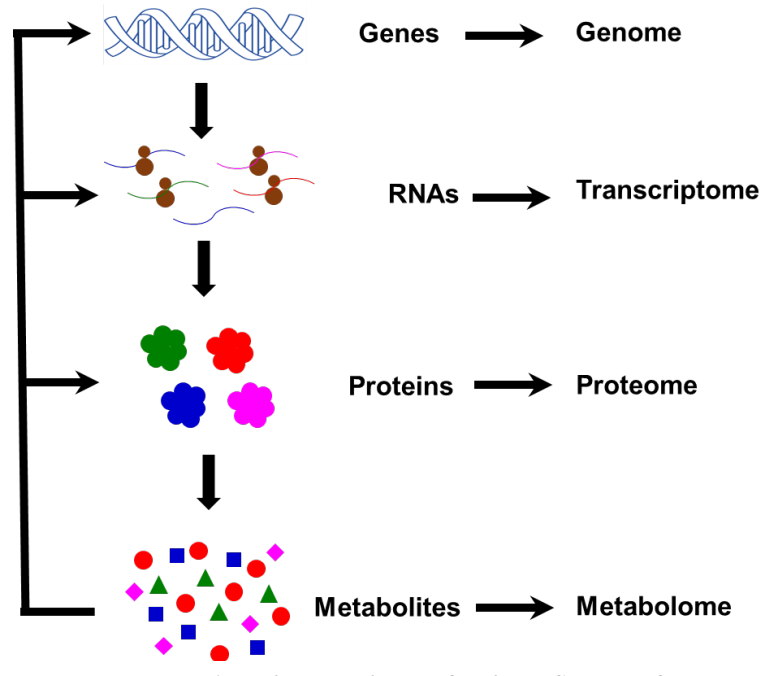

Figure 1. The hierarchy of the flow of genetic information. Genes, which make up the organism's genome, encode the various ribonucleic acid (RNA) species (transcriptome). Some RNAs are translated to proteins. The activity of these proteins and the influence of exogenous factors (e.g. diet), determines the metabolite inventory (metabolome) of a cell, tissue, or biological fluid. The metabolites, in turn, can affect gene expression, enzyme activities, and protein functions.

to simultaneously measure as many metabolites as possible from biological samples without bias. It involves comparing the metabolome of experimental and control samples to spot differences between their metabolite profiles. On the other hand, targeted metabolomics focuses on validation, wherein large numbers of samples are used to explain the effect of natural and biological variations in validating the identity of the previously determined metabolites. In this case, it requires high certainty of identification. Both approaches have been widely used in various BM metabolomics studies as described in the following sections.

As comprehensive BM metabolomics research is primarily driven by the advancements in instrumentation and analytical techniques, the present review article aims to discuss current and developing methodologies used in BM metabolomics. We then summarized recent investigations into the BM metabolome that benefited from these methodological and analytical developments. In an attempt to highlight the role of BM metabolomics as a tool to bridge the gap between mother phenotype and/or nutrition and infant health outcomes, the influence of maternal nutrition and phenotype on specific BM metabolites and the impact of some BM metabolites on infant nutrition and health outcomes are explored. Lastly, the typical workflow and strategies on BM metabolomic data analysis and some recent advances in BM metabolomics are described. 


\section{INSTRUMENTATIONS USED IN BREASTMILK METABOLOMICS}

Metabolomic studies often require large number of samples, hence, the demand for high-throughput approaches. Nuclear magnetic resonance (NMR) spectroscopy and mass spectrometry (MS)-based approaches represent the most popular choice of instrumentation for BM metabolomic studies. A comprehensive review of these techniques and their advantages and disadvantages were discussed in a previous study (Wishart, 2008).

High resolution ${ }^{1} \mathrm{H}-\mathrm{NMR}$ spectroscopy has been an attractive technique in both targeted and untargeted BM metabolomics. With most compounds having hydrogen, ${ }^{1} \mathrm{H}-\mathrm{NMR}$ provides a robust approach in the detection and identification of various metabolites (Savorani et al., 2013), thus several BM metabolomic studies have employed this technique (Smilowitz et al., 2013; Praticò et al., 2016; Longini et al., 2014; Sundekilde et al., 2016; Wu et al., 2016). Unlike MS-based techniques, NMR is nondestructive allowing preservation of metabolites for multiple analysis (Ryan et al., 2013). One of the strengths of NMR-based metabolomics is that it can provide structural and quantitative information on the metabolite which facilitates identification of unknown metabolites, a major hurdle in metabolomics (Brennan, 2016). A major drawback of NMR-based techniques is the lack of sensitivity making detection of low abundance metabolites difficult (Brennan, 2016; Sundekilde et al., 2016).

MS-based techniques are extremely sensitive especially when combined with separation methods, such as gas chromatography (GC) and liquid chromatography (LC). The type of separation depends on the investigator's objective. GC-MS is commonly used for the detection of nonpolar, volatile or semi-volatile metabolites such as fatty acids and a range of polar metabolites such as amino acids, and tricarboxylic acid (TCA) cycle and glycolysis intermediates (Brennan, 2016). GC-MS also permits detection of other metabolites such as steroids, diglycerides, mono-, di- and trisaccharides, and sugar alcohols. A major limitation of GC-MS is that metabolites must be volatile or amenable to chemical derivatization to permit detection. LC-MS is commonly used for the detection of polar, nonvolatile metabolites such as amino acids, sugars, sugar alcohols, organic acids, organic amines, and nucleotides. Therefore, LC-MS has a wider coverage of metabolites than GC-MS and has been widely used in discovery-based approaches or when targeted approaches fail to detect metabolites because of volatility issues.
Due to the inherent complexity of BM composition, a truly comprehensive metabolomics research requires the combined strengths of NMR and MSbased techniques. Several factors can contribute to the extent of comprehensiveness of metabolomics experiments such as the chemical nature of metabolites, sample matrix, and cost of analysis, which will then influence the choice of methodology.

\section{RECENT INVESTIGATIONS INTO THE BREASTMILK METABOLOME}

Despite advances in instrumentations and methodologies used in metabolomics, relatively few studies have attempted to unravel the metabolome of BM. Three of these studies are summarized in Table 1. Using high-resolution ${ }^{1} \mathrm{H}-\mathrm{NMR}$ and GCMS, Marincola et al. (2012) identified differences in metabolite composition between pre-term human BM and infant formula milk (FM). In this study, lactose was found to be higher in pre-term BM compared to FM. On the other hand, FM was shown to have higher levels of maltose compared to pre-term BM. FM was also found to contain higher levels of oleic and linoleic acids than BM. However, the few number of samples involved in the study may not warrant a strong conclusion on the observed altered metabolites between human BM and FM. Nevertheless, the findings reinforce the great potential of NMR spectroscopy in discriminating metabolites between pre-term BM and FM which may have important implications in improving the nutritional quality of formula milk intended for preterm infants.

Using ${ }^{1} \mathrm{H}-\mathrm{NMR}$ spectroscopy, Sundekilde et al. (2016) identified differences in BM metabolite profiles in women of different gestational and lactational ages. The levels of valine, leucine, betaine, and creatinine were found to be elevated in colostrum from term mothers compared with mature milk. On the other hand, the levels of glutamate, caprylate, and caprate were increased in mature term milk compared to colostrum. Pre-term colostrum exhibited higher levels of oligosaccharides, citrate, and creatinine while BM from women postpartum had increased levels of valine, leucine, glutamate, and pantothenate. The study further indicates that marked differences in specific metabolites exist in BM between mothers delivering pre-term and fullterm. Furthermore, temporal changes in metabolite composition were observed in pre-term $\mathrm{BM}$ to resemble that of full-term BM.

In a study conducted by Qian et al. (2016), GCTOFMS and UPLC-QTOFMS were used to compare the metabolite composition between human BM, FM, and bovine milk (BovM). This 
study revealed that human BM generally had higher levels of non-esterified saturated, monounsaturated, and polyunsaturated fatty acids (SFA, MUFA, and PUFA, respectively) compared with FM and BovM. The levels of some free amino acids are also higher in human BM compared with FM and BovM. Furthermore, human BM exhibited lower levels of tricarboxylic acid cycle (TCA) intermediates compared with FM and BovM. These findings indicate that each of the milk type studied has a unique metabolite profile and that there are metabolites that are characteristically higher in human BM compared to FM (or BovM). Therefore, this may aid in the development of strategies to improve the quality of formula milk to support optimal health and development of infants who have little or no access to BM.

\section{EFFECTS OF MATERNAL NUTRITION AND PHENOTYPE ON BREASTMILK METABOLITES}

A number of studies have demonstrated that the compositional variability of BM is highly influenced by maternal dietary intake and phenotype. For example, BM FAs are derived from mammary gland FA synthesis and the FAs from the maternal plasma (Innis, 2014), which are both influenced by maternal nutrition (Innis, 2014). An extensive body of literature from different countries showed that many of the fatty acids in human BM, including MUFAs, omega-3 and -6 FAs are strongly influenced by maternal lipid nutrition (Innis, 1992; Koletzko et al., 1992; Koletzko et al., 1999; Novak et al., 2011). In a study by Koletzko et al (1992), MCFAs were shown to be elevated in lactating women with high carbohydrate, low-fat diets and low plasma triglycerides. However, a recent study suggests that triglyceride secretion into human BM is modulated by the inverse regulation of fatty acid synthesis in the mammary gland and the uptake of FAs from maternal plasma, regardless of the dietary fat or carbohydrate intake (Novak et al., 2011). Therefore, it remains to be investigated how maternal nutrition influences the dynamics of various types of lipids in BM.

Francois et al. (1998) investigated the bioavailability of marker FA in BM after maternal ingestion. Test formulas containing different oils with characteristically high amounts of specific FA, such as coconut oil (high in lauric acid), where fed to lactating women periodically. BM was shown to have elevated levels of these FA only 6 hours after consumption. Marker FA levels peaked at 10-14 hours; two notable exceptions peaking at 72 hours post-consumption, eicosapentanoic acid (EPA) and docosahexanoic acid (DHA), are both found in fish products.
Long-term effects of a vegetarian and vegan maternal diet on the FA composition of BM have also been studied (Sanders et al., 1992). It was observed that BM from vegetarian and vegan mothers exhibited higher amounts of linoleic acid (LA) yet lower amounts of (DHA). It was concluded that these variations in FA composition can be attributed to maternal diet, as omnivorous mothers are supplied a normal amount of DHA whereas vegan mothers have higher LA consumption.

There are relatively few studies that employed a metabolomic approach to examine the effects of maternal nutrition and phenotype in BM composition. In one study, milk collected at day 90 postpartum from 52 healthy women was analyzed by using ${ }^{1} \mathrm{H}-\mathrm{NMR}$ spectroscopy (Smilowitz et al., 2013). A total of 65 metabolites were detected. The investigators indicate that human milk metabolome is relatively insensitive to fluctuations in health or diet for relatively healthy individuals. It was noted, however, that maternal BMI measured at day 60 postpartum is negatively correlated with BM acetone levels out the 65 metabolites detected in BM.

An NMR-based metabolomic study of BM composition identified 43 different metabolites, from which 21 are fucosylated human milk oligosaccharides (fuc-HMOs) (Practico et al., 2014). The study revealed differences in HMO fucosylation patterns in BM which may be affected by two main enzymes, $\alpha-1,2$-fucosyltransferase (FucT2) and $\alpha$-1,4-fucosyltransferase (FucT4) (Totten et al., 2012). FucT2 is encoded by the secretor (Se) gene while FucT4 is encoded by the Lewis blood group gene (Le). Four possible maternal phenotypes arise from the level of expression of these two genes, $\mathrm{Se}+1$ $\mathrm{Le}+, \mathrm{Se}+/ \mathrm{Le}-, \mathrm{Se}-/ \mathrm{Le}+, \mathrm{Se}-/ \mathrm{Le}$. The study showed marked variations in metabolite profiles between two maternal phenotypes ( $\mathrm{Se}+/ \mathrm{Le}+$ vs $\mathrm{Se}-/ \mathrm{Le}+$ ). These findings suggest that the variability of fucHMO in BM is highly dependent on the maternal phenotype. With accurate diagnosis, these findings may warrant inclusion of fuc-HMOs in the infant's diet to support gut health.

\section{IMPACT OF BREASTMILK ON INFANT HEALTH OUTCOMES}

The changing BM composition is attuned, at least in part, to the infant's changing nutritional and physiological demands. In addition, BM contains natural bioactive compounds that positively contribute to the metabolic, immune, and gut microbiome development of the breastfed infant which may influence long-term health outcomes (Villaseñor et al., 2014). 
Table 1. Summary of Recent Metabolomic Studies in Breastmilk.

\begin{tabular}{|c|c|c|c|c|c|}
\hline $\begin{array}{l}\text { Aim of the } \\
\text { Study }\end{array}$ & $\begin{array}{l}\text { Study } \\
\text { Participants } \\
\text { or Samples } \\
\text { Collected }\end{array}$ & $\begin{array}{l}\text { Analytical } \\
\text { Platform }\end{array}$ & $\begin{array}{l}\text { Identified and/or Altered } \\
\text { Metabolites }\end{array}$ & $\begin{array}{l}\text { Observed } \\
\text { Trend }\end{array}$ & $\begin{array}{l}\text { First Author } \\
\text { and Year }\end{array}$ \\
\hline $\begin{array}{l}\text { The metabolite } \\
\text { profile of BM } \\
\text { from mothers } \\
\text { delivering } \\
\text { pre-term was } \\
\text { investigated and } \\
\text { compared with } \\
\text { formula milk. }\end{array}$ & $\begin{array}{l}\text { Mothers } \\
\text { delivering pre- } \\
\text { term infants } \\
(\mathrm{n}=20) \\
\text { Formula milk } \\
(\mathrm{n}=13)\end{array}$ & $\begin{array}{l}{ }^{1} \mathrm{H}-\mathrm{NMR} \\
\text { GC-MS }\end{array}$ & $\begin{array}{l}\text { Lactose } \\
\text { Maltose } \\
\text { Oleic and linoleic acid }\end{array}$ & $\begin{array}{l}\uparrow \text { in PTM vs FM } \\
\downarrow \text { in PTM vs FM } \\
\downarrow \text { in PTM vs FM }\end{array}$ & $\begin{array}{l}\text { Marincola C } \\
\text { (2012) }\end{array}$ \\
\hline $\begin{array}{l}\text { The metabolite } \\
\text { profiles of BM } \\
\text { from mothers } \\
\text { delivering } \\
\text { pre-term and } \\
\text { full-term were } \\
\text { characterized and } \\
\text { compared. }\end{array}$ & $\begin{array}{l}\begin{array}{l}\text { Mothers } \\
\text { delivering pre- } \\
\text { term infants } \\
(\mathrm{n}=15)\end{array} \\
\text { Mothers } \\
\text { delivering full- } \\
\text { term infants } \\
(\mathrm{n}=30)\end{array}$ & ${ }^{1} \mathrm{H}-\mathrm{NMR}$ & $\begin{array}{l}\text { Fucosylated oligosaccharides } \\
\text { fucose, N-acetylneuraminic acid, } \\
\mathrm{N} \text {-acetylglucosamine } \\
\text { Val, Leu, pantothenate, citric acid, lactic } \\
\text { acid, betaine, and creatinine } \\
\text { Glutamate, butyrate, caprylate, and caprate } \\
\text { Citrate, lactose, and phosphocholine } \\
\text { Carnitine, caprylate, caprate, pantothenate, } \\
\text { beta-hydroxybutyrate, and urea } \\
\text { Lactose, fucosyl moieties, } \mathrm{N} \text {-acetylneur- } \\
\text { aminic acid, N-acetylglucosamine, 3'-si- } \\
\text { alyllactose, 6'-sialyllactose, lacto-N-difuco- } \\
\text { hexaose I, Glu, citric acid, phosphocholine, } \\
\text { choline, and formic acid }\end{array}$ & $\begin{array}{l}\uparrow \text { in colostrum vs } \\
\text { MM } \\
\uparrow \text { in colostrum and } \\
\text { TM vs MM } \\
\uparrow \text { in MM vs } \\
\text { colostrum and TM } \\
\uparrow \text { in PTM vs FT } \\
\text { milk } \\
\downarrow \text { in PTM vs FTM } \\
\uparrow \text { in PTM vs FTM }\end{array}$ & $\begin{array}{l}\text { Sundekilde U } \\
\text { (2016) }\end{array}$ \\
\hline $\begin{array}{l}\text { The metabolome } \\
\text { of HM, BovM, } \\
\text { and FM was } \\
\text { compared. }\end{array}$ & $\begin{array}{l}\text { Lactating women } \\
(\mathrm{n}=30) \\
\text { BovM }(\mathrm{n}=20) \\
\text { FM }(\mathrm{n}=20)\end{array}$ & $\begin{array}{l}\text { GC-TOF-MS } \\
\text { UPLC-QTOF- } \\
\text { MS }\end{array}$ & 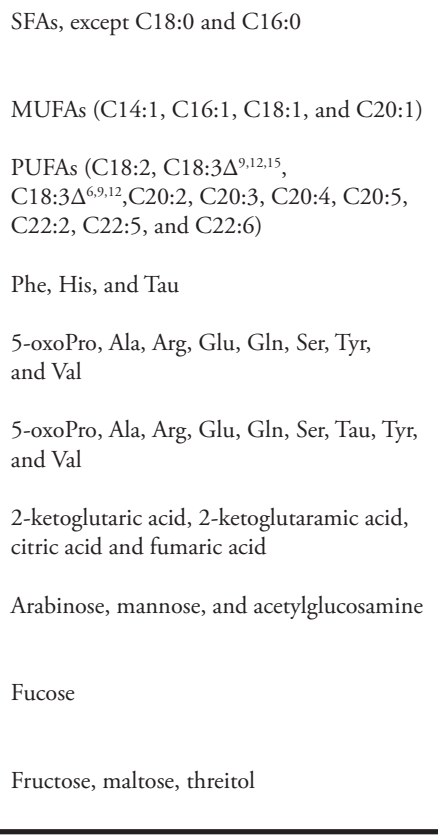 & $\begin{array}{l}\uparrow \text { in HM vs BovM } \\
\text { and FM } \\
\uparrow \text { in HM vs BovM } \\
\text { and FM } \\
\uparrow \text { in HM vs BovM } \\
\text { and FM } \\
\downarrow \text { in HM vs FM } \\
\uparrow \text { in HM vs FM } \\
\uparrow \text { in HM vs BovM } \\
\downarrow \text { in HM vs FM } \\
\downarrow \text { in HM vs FM and } \\
\text { BovM } \\
\uparrow \text { in HM vs FM and } \\
\text { BovM } \\
\downarrow \text { in HM and BovM } \\
\text { vs FM }\end{array}$ & Qian L (2016) \\
\hline
\end{tabular}

Abbreviations: $M M$, mature milk; PTM, pre-term milk, FT, full-term milk; HM, human milk; FM, formula milk; BovM, bovine milk; SFA, saturated fatty acid; MUFA, monounsaturated fatty acid; PUFA, polyunsaturated fatty acid; C16:0, palmitic acid; C18:0, stearic acid; C14:1, myristoleic acid; C16:1, palmitoleic acid; C18:1, oleic acid; C20:1, eicosenoic acid;

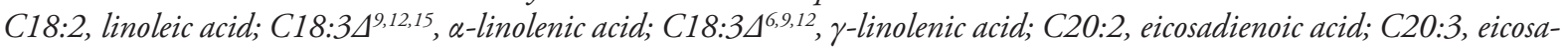
trienoic acid; C20:4, arachidonic acid; C20:5, eicosapentaenoic acid; C22:2, docosadienoic acid; C22:5, docosapentaenoic acid; C22:6, docosahexaenoic acid; Val, valine; Leu, leucine; Phe, phenylalanine; His, histidine; Tau, taurine; 5-oxoPro, 5-oxoproline; Ala, alanine; Arg, arginine; Glu, glutamic acid; Gln, glutamine; Ser, serine; Tyr, tyrosine. 
In a recent study, the urine samples of two groups of infants, breastfed and formula-fed, were analyzed using capillary electrophoresis-time-of-flight (CETOF) MS (Shoji et al., 2017). The study showed that urinary choline metabolites (choline base solution, $N, N$-dimethylglycine, sarcosine, and betaine) at 1 month were higher in breastfed infants than in formula-fed infants. Breastfed infants exhibited lower levels of urinary lactic acid at 1 month and 6 months compared with formula-fed infants. Breastfed infants also exhibited lower levels of urinary threonine and carnosine at 1 month compared with formula-fed infants. These findings indicate that the type of feeding during early infancy can influence the infant's choline metabolism, as well as the levels of other metabolites. This study agrees well with Ilcol et al. (2005) study which showed the direct correlation between infant serum choline levels and their mother's BM choline content. Serum free choline levels were shown to decrease as phospholipid-bound choline increased over time. Both forms of choline were found to be doubled in breast-feeding women 12-28 post-partum. In an earlier study, it was determined that the total choline content in human colostrum is lower than in mature milk seven days post-partum, which coincides with the acceleration in growth in neonates at this stage (Holmes et al., 2000). Choline is an important metabolite for infant development due to its role in brain development and function (Zeisel et al., 1994). An increased risk of birth defects was also observed on infants with low choline intake (Shaw et al., 2004).

The role of various BM lipids on the growth and development of infants has been studied for many years. In a study by Xiang et al. (2000), the BM fatty acid composition and the growth rate of the infant brain were analyzed. The study showed that the ratio between arachidonic acid (AA) and DHA in BM was positively correlated with the rate of gain of the occipito-frontal head circumference and the calculated brain weight at 1 month and 3 months of age, respectively. However, the exact requirements for $\mathrm{AA}$ and DHA for optimal growth and neurodevelopment of infants remain to be established.

Aside from common metabolites such as sugars, amino acids and fatty acids, dietary polyphenols, such as epicatechin were shown to be accessible to infants through their mother's BM (Khymenets et al., 2016). Using solid-phase extraction-ultra performance liquid chromatography-tandem mass spectrometry (SPE-UPLC-MS/MS) for direct epicatechin metabolite analysis, several host and and microbial phase II metabolites of epicatechin were detected in mother's BM after a single dose of dark chocolate. These findings suggest that the coordinated action of an infant's metabolism and gut microbial processing promotes bioavailability of epicatechin from the mother's BM. Importantly, further studies are needed on the role of BM antioxidants on infant's growth and development in light of the benefits of polyphenols which are known to be effective anti-oxidants and anti-inflammatory agents (Landete, 2012).

BM metabolomics is still in its early stage of development locally. In our previous study, BM and plasma retinol of lactating women who participated in the Sixth Philippine National Nutrition Survey were measured by high-performance liquid chromatography (HPLC) (Sumayao et al., 2009). The study showed that BM retinol levels were highest in women at their first 3 months of lactation and remained constant thereafter up to 11 months. Breastfed infants whose mothers have normal BM retinol levels also displayed higher plasma retinol levels compared with breastfed infants whose mothers have low BM retinol levels. This study indicates that BM retinol levels change over the period of lactation and can influence the vitamin A status of breastfed infants. Postpartum maternal vitamin A supplementation, exclusive or frequent breastfeeding for the first 6 months, and addition of complementary foods rich in vitamin A by 6 months are recommended to achieve optimal benefits for the infant.

Several metabolite components of BM also play important roles in forming an ideal gut bacterial community for neonates. A growing body of evidence points to the impact of HMOs in orchestrating the infant's gut microbiome. High concentrations of HMOs $(10-15 \mathrm{~g} / \mathrm{L})$ are delivered to infants through their mothers' BM (Smilowitz et al., 2013). These oligosaccharides are made from five monosaccharides: glucose, galactose, $\mathrm{N}$-acetylglucosamine, fucose, and sialic acid. Roughly 200 HMOs of varying chain lengths are currently known. These oligosaccharides are not affected by enzymatic hydrolysis in the upper gastrointestinal tract (Engfer et al., 2000). Hence, they are not utilized for energy consumption and may serve a different function in infant's health.

In the past years, studies have emerged supporting claims that short-chain HMOs promote the growth of the enteric genus, Bifidobacteria. HMOs' prebiotic effects have since been known. A study by LoCasio et al. (2007) suggests that some enzymes on the bacterial cell membrane are only triggered by $\mathrm{HMOs}$ of specific lengths and states. HMO profiling shows that one strain of bifidobacteria favors $63.9 \%$ of total HMOs. This strain, Bifidobacterium longum bivar infantis, was also shown to contain fucosidases and sialidases. These enzymes enable the gut microbiota 
in effectively utilizing fuc-HMOs and sia-HMOs. Despite extensive studies on the prebiotic effects of HMOs on the gut microbiota of infants, this does not explain the fact that $90 \%$ of consumed and unaltered HMOs are excreted through faecal matter (Bode, 2009). This suggests that the function of HMOs extend beyond creating an ideal gut bacterial community in infants.

Potentially pathogenic bacteria such as Escherichia coli, Vibrio cholera, and Campylobacter jejuni are known to bind to intestinal epithelial cells via their receptors that recognize oligosaccharides on the surface of these cells (Sharon, 1996). HMOs display structural similarities with cell surface-associated oligosaccharides, thus facilitating disruption of potentially pathogenic invasions. HMOs, at high concentrations, were shown to directly attach onto the bacterial cell surface through its receptors which then blocks the pathogen's attachment to intestinal epithelial cells (Newburg et al., 2005). In a study by Ruiz-Palacios et al. (2003), fuc-HMOs prevented Campylobacter adherence in mice in vivo and in human intestinal cells ex vivo. Mouse pups were shown to have Campylobacter resistance when their mothers had been transfected with a human $\alpha-1,2$-fucosyltransferase gene which promotes production of fuc-HMOs in BM which inhibits Campylobacter adherence to intestinal mucosa. This mechanism blocks the critical step in Campylobacter's pathogenicity.

These studies highlight the protective effects of a distinct group of metabolites in BM against potentially pathogenic bacteria. However, the role of HMOs on the infant's gut microbiome merits further investigation to realize its full benefits in the overall health of infants. Nevertheless, it is certain that breastmilk remains as an unparalleled biofluid through which an infant's gut microbiota is orchestrated as well as its means to sufficiently sustain a healthy gut bacterial community.

A study by Hong et al. (2009) suggests that HMOs may prevent HIV-1 transmission from mother to child. The HIV-1 viral envelope contains a glycoprotein, gp120, which binds to dendritic cellspecific ICAM3-grabbing non-integrin (DC-SIGN), a protein on the surface of dendritic cells ( $\mathrm{Su}$ et al., 2003). Once the virus is captured, it hides inside the DC and escapes before the body's immune system recognizes the virus. This phenomenon triggers HIV-1 infection. DC-SIGN has an affinity towards mannose-containing glycans, the characteristic oligosaccharides present in viral gp120 (van Liempt et al., 2006). However, it has a greater affinity to glycans containing a fucosylated N-LacNAc residue, a characteristic oligosaccharide also present in $\mathrm{BM}$
HMOs. Using in vitro assays, it was shown that that physiological concentrations of HMOs markedly lowered attachment of gp120-containing HIV-1 to DC-SIGN by $>80 \%$. These findings suggest a novel role of distinct group of BM HMOs as protective agents against mother-to-infant HIV transmission and can potentially lead to the development of strategies to protect breastfed infants from HIV infection from HIV-positive mothers.

As evidenced by the cited studies, various metabolites in BM have specific roles in the proper growth and nurturing of neonates. Despite the advances in BM metabolomics, there is a demand for substantial research to reveal other benefits of BM metabolites on infant health outcomes in order to develop strategies aimed at promoting ideal feeding practices for neonates. These findings only fortify the statement 'breast milk is the gold standard for infant nutrition.

\section{BREASTMILK METABOLOMIC DATA ANALYSIS}

Various bioinformatics tools and techniques have been developed for the analysis of metabolomics data (Bartel et al., 2013; Korman et al., 2012). The present review will focus on the typical workflow used in discovery metabolomics which has been widely applied in BM metabolomics data sets.

Discovery metabolomics traditionally involves four major steps as shown in Figure 2; (1) metabolite profiling, (2) metabolite identification, (3) statistical analysis, and (4) data interpretation. Profiling involves identification of potentially interesting metabolites that are differentially regulated between the experimental and control samples. It is essential to find as many metabolites (i.e. feature finding) as possible. For LC- or GC-MS analysis, feature finding must be equipped with a deconvolution program which aids in reconstructing a single component spectrum for each metabolite that is chromatographically unresolved or poorly resolved with co-eluting metabolites. In LC- or GC-MS output, a metabolite is represented by its molecular features, which are defined by the combination of retention time, mass or mass spectra and abundance. It is not necessary to determine the identity of the metabolites during the profiling step. However, the molecular features of each metabolite will facilitate tracking of metabolites in between sample runs which, in turn, aids in the assessment of reproducibility of the analytical technique used in metabolite detection.

One-dimensional high-resolution ${ }^{1} \mathrm{H}-\mathrm{NMR}$ spectroscopy is the most widely used NMR approach 


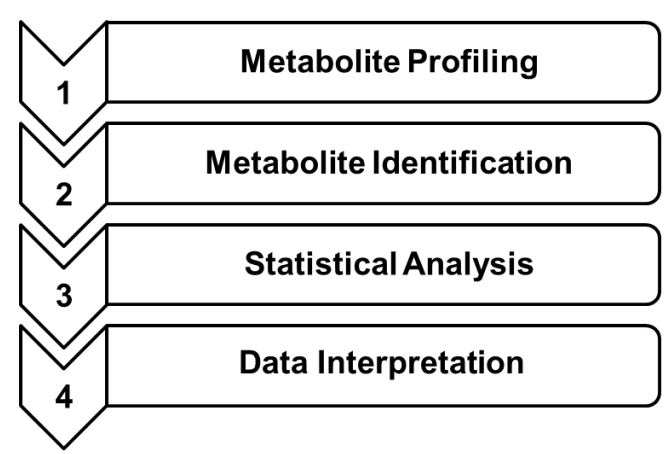

Figure 2. Typical Workflow of Breastmilk Metabolomic Data Analysis.

in BM metabolomics (Sundekilde et al., 2013). Metabolite profiling using ${ }^{1} \mathrm{H}-\mathrm{NMR}$ involves detection of chemically distinct hydrogen nucleus in each metabolite in a biological fluid, such as BM, that exhibits a NMR signal at a characteristic resonance frequency, which is measured as a chemical shift to a standard compound. Each metabolite is characterized by an exact chemical shift of the NMR signal of its hydrogen nucleus and is independent of the applied field strength and the particular matrix conditions.

High quality electron ionization spectra are essential for positive identification of metabolites. Metabolites that are separated and detected by either LC- or GCMS are characterized by comprehensive molecular features such as mass, retention time, and relative abundance. These features are used to identify the metabolite using commercial metabolomic libraries or publicly available databases such as the HMDB (Wishart et al., 2007).

In ${ }^{1} \mathrm{H}-\mathrm{NMR}$ spectroscopy, the experimental chemical shifts of hydrogens in metabolites are either binned and then analyzed or fitted to patterns of signals corresponding to the metabolites expected to be present in the biofluid. As in LC- or GCMS, identification of metabolites using ${ }^{1} \mathrm{H}-\mathrm{NMR}$ spectroscopy can performed by comparison with annotated metabolites using commercial metabolomic libraries or publicly available databases such as the Human Metabolome Database (HMDB) (Wishart et al., 2007). One of the strengths of NMR spectroscopy is that the chemical shifts are very sensitive to structural or environmental changes, hence can distinguish or identify metabolites in complex matrices and can decrease the likelihood of two similar molecules having identical ${ }^{1} \mathrm{H}-\mathrm{NMR}$ spectra (Dona et al., 2016).

NMR and MS-based metabolomic data sets consist of a large number of samples and variables. Therefore, a number of preprocessing steps must be performed in order to obtain a reliable data suitable for downstream data analysis. This may include data alignment, data reduction (binning), normalization, and scaling. Metabolomic statistical analysis typically starts with unsupervised data analysis which allows the researcher to visualize the internal structure of the data and to gain insights into the underlying trend between experimental groups under study (Brennan, 2016). Univariate and multivariate statistical analysis represent two traditional statistical analysis used widely in the analysis of metabolomics data sets. Classical univariate methods include t-test and analysis of variance (ANOVA) which are commonly used to compare means or medians of one variable across two or more groups (Xia and Wishart, 2007). Multivariate approaches involve simultaneous analysis of more than two variables. An example of a multivariate method is principal component analysis (PCA). PCA represents an unsupervised clustering or classification method commonly used in preliminary metabolomic data analysis (Brennan, 2016). It projects complex, multi-dimensional data into a coordinate system with fewer dimensions (also called principal components) (Xia and Wishart, 2007). PCA aims to maximize the data variance in the first principal components of metabolite features (Xia \& Wishart, 2007). Hence, PCA has been routinely used by metabolomics researchers to identify outlier features in complex data sets. However, caution should be observed when reporting PCA models as there is no guarantee that the directions of maximum variance will contain the best features for discrimination among experimental groups.

The last step in the workflow facilitates connection between the various differentially expressed metabolites and the relationship of these metabolites to biological processes, also known as pathways. Pathway analysis helps elucidate the biological relevance of complex metabolomics data sets in a systems context. MetaboAnalyst is a freeware that is equipped with a high-level functional interpretation for pathway enrichment and pathway topology analysis which supports 15 model organisms with -1,200 precompiled Kyoto Encyclopedia of Genes and Genomes (KEGG) (Xia and Wishart, 2007). An example of pathway analysis generated from identified metabolites in breastmilk is shown in a previous study (Villaseñor et al., 2014).

The success of metabolomics data analysis relies upon the analytical reproducibility of the methodologies and instrumentations employed in metabolite detection and the quality of the mass data used in metabolite identification. Because metabolomes may display natural variations even in normal or healthy subjects, it is imperative that a sizeable number of subjects or samples be used to generate biologically meaningful data. This requires analytical 
instruments used in metabolomics data acquisition to have high sensitivity and accuracy and to operate on high throughput.

\section{RECENT ADVANCES IN BREASTMILK METABOLOMICS}

Various methodologies and techniques have been developed to improve the accuracy, sensitivity, robustness, and coverage of metabolite identification in BM. One of the hurdles in BM metabolomics is the requirement for separate metabolite extraction procedures to capture both polar and lipidic compounds in BM. A recent technique has been developed to capture multiple classes of metabolites using a single extraction phase followed by GC-MS or LC-MS analysis (Villaseñor et al., 2014). This is a significant improvement from pre-existing twophase methods since it is more efficient in terms of time and cost, as well as serving two different analytical platforms. The method was used to comprehensively characterize the human BM metabolome and identify multiple interconnected pathways with the aid of pathway mapping tools. In most cases, metabolites from specific pathways can be identified using a single technique only. However, there are some metabolic pathways that require both GC-MS and LC-MS for identification; examples include pathways that are co-regulated by gut bacteria (Villaseñor et al., 2014).

More recently, the most comprehensive metabolite profile of BM to date has been mapped using four different analytical platforms (HPLC-MS and ultra-performance LC-MS, GC-MS, CE-MS, and ${ }^{1} \mathrm{H}-\mathrm{NMR}$ spectroscopy) and two sample preparation techniques (modified Folch extraction and single phase extraction method) (Andreas et al., 2015). This yielded a total of 710 metabolites spanning multiple classes of metabolites such as lipids, amino acids, aromatics, sugars, HMOs, organic acids. With regards to the two sample preparation techniques, the single phase extraction followed by HPLC-MS analysis gave the best profile but did not capture the HMO, amino acids, and sugars. Furthermore, the analytical strategies performed revealed temporal changes in some BM metabolites which are known to be important for infant immunological, neurological, and gastrointestinal development. This demonstrates the utility of a multiplatform analytical technique to investigate questions of clinical and biological significance of BM metabolites as well as assessing the impact of environmental and dietary factors on the composition of BM, with emphasis on the downstream consequences these may have on infant's health.

\section{FUTURE DIRECTIONS AND CONCLUSIONS}

Previous metabolomics studies performed on BM explored the relationship between the metabolome, as well as the mother's diet, lifestyle, phenotype and the infant's health outcomes. Although still in its incipient stages, BM metabolomics has shown great potential as a key technology to improve our understanding of the inherent compositional variability of BM. High quality metabolomics data could be linked to known and validated clinically relevant disease conditions and must be considered for the development of biomarkers for translation to the clinical setting. Using these 'metabolite signatures' and their involvement in various metabolic pathways, functional networks can be built to allow researchers to gain deeper insights into the mechanistic events that lead to various physiological imbalances.

In obtaining a complete picture of the biological state of an organism, metabolomics data can be integrated with genomics and proteomics data. Using integrative pathway analysis from multi-omics data sets, it is possible to find connections between dysregulated metabolites in $\mathrm{BM}$ and the relevant genes and proteins that regulate their circulating levels. Based on these pathways, functional networks can be constructed to reveal the full spectrum of the metabolite's biological function and specific pathophysiological events. In addition, integrative omics data can be utilized to tailor dietary needs of an individual for disease prevention, also known as personalized nutrition. Importantly, these distinctive metabolites may identify therapeutic targets for disease prevention or may allow treatment strategies to be developed in the pipeline.

In conclusion, BM metabolomics has provided invaluable information on the presence multiple biochemical factors in BM that can influence in infant nutrition, development, and health outcomes. Furthermore, BM metabolomics has revealed that maternal diet, phenotype and various clinical conditions can strongly influence the compositional variability of BM and can possibly unravel mechanistic links in the chemical interaction between the lactating mother and breastfed infant. Furthermore, the existence of non-nutritional factors and bioactive compounds in human BM and their potential benefits in infant health, especially in gut microbiome development and as a source of essential secondary metabolites, warrant further investigation to realize the full benefits of $\mathrm{BM}$ consumption and/or exclusive breastfeeding among infants. Importantly, identification of these biochemical factors in BM may form a critical component in 
formulating dietary interventions targeting lactating women and in promoting strategic feeding practices among breastfed infants and may also benefit infants with little or no access to BM by improving the formulation of their complementary food or formula milk. These advances are vital in the future development of pediatric research.

\section{REFERENCES}

Allen J, Hector D. Benefits of breastfeeding. N S W Public Health Bull 2005;16(4):42-6. Available from: http://phrp.com.au/issues/volume-16-issue-3-4/ benefits-of-breastfeeding doi: 10.1071/NB05011.

Andreas NJ, Hyde MJ, Gomez-Romero M, LopezGonzalvez MA, Villaseñor A, Wijeyesekera A, et al. Multiplatform characterization of dynamic changes in breast milk during lactation. Electrophoresis 2015;36(18):2269-2285. Available from: http://doi. wiley.com/10.1002/elps.201500011 doi: 10.1002/ elps.201500011.

Astarita G, Langridge J. An emerging role for metabolomics in nutrition science. J Nutrigenet Nutrigenomics 2013;6(4-5):181-200. Available from: https://www.karger.com/Article/ FullText/354403 doi: 10.1159/000354403.

Bartel J, Krumsiek J, Theis FJ. Statistical methods for the analysis of high-throughput metabolomics data. Comput Struct Biotechnol J 2013;4(5):1-9. doi: $10.5936 /$ csbj.201301009.

Bode L. Human milk oligosaccharides: prebiotics and beyond. Nutr Rev 2009;67(2). doi: 10.1111/j.17534887.2009.00239.x.

Brennan L. Metabolomics in nutrition research-a powerful window into nutritional metabolism. Essays Biochem 2016;60(5):451-8. Available from: http://essays.biochemistry.org/cgi/doi/10.1042/ EBC20160029 doi: 10.1042/EBC20160029.

Dona AC, Kyriakides M, Scott F, Shephard EA, Varshavi D, Veselkov $\mathrm{K}$, et al. A guide to the identification of metabolites in NMR-based metabonomics/metabolomics experiments. Comput Struct Biotechnol J. 2016 2016;14:135153. Available from: http://linkinghub.elsevier.com/ retrieve/pii/S2001037015300192 doi: 10.1016/j. csbj.2016.02.005.

Engfer M, Stahl B, Finke B, Sawatzki G, Daniel $\mathrm{H}$. Human milk oligosaccharides are resistant to enzymatic hydrolysis in the upper gastrointestinal tract. Am J Clin Nutr 2000:1589-1596.
Francois CA, Connor SL, Wander RC, Connor WE. Acute effects of dietary fatty acids on the fatty acids of human milk. Am J Clin Nutr 1998;67:301-308.

Holmes HC, I. Snodgrass, G. J. A, , Iles RA. Changes in the choline content of human breast milk in the first 3 weeks after birth. European Journal of Pediatrics 2000;159(3):198-204. Available from: http://link.springer.com/10.1007/s004310050050 doi: $10.1007 / s 004310050050$.

Hong P, Ninonuevo M, Lee B, Lebrilla C, Bode L. Human milk oligosaccharides reduce HIV-1-gp120 binding to dendritic cell-specific ICAM3-grabbing non-integrin (DC-SIGN). Br J Nutr 2009:482-486.

Horta BL, Victora CG. Long-term effects of breastfeeding-a systematic review. World Health Organization 2013.

Horta BL, Victora CG. Short-term effects of breastfeeding: a systematic review on the benefits of breastfeeding on diarrhoea and pneumonia mortality. World Health Organization 2013.

Ilcol YO, Ozbek R, Hamurtekin E, Ulus IH. Choline status in newborns, infants, children, breast-feeding women, breast-fed infants and human breast milk. J Nutr Biochem 2005;16(8):489-99. Available from: http://linkinghub.elsevier.com/ retrieve/pii/S095528630500029X doi: 10.1016/j. jnutbio.2005.01.011.

Innis SM. Human milk and formula fatty acids. J Pediatr 1992;120:S56-61.

Innis SM. Impact of maternal diet on human milk composition and neurological development of infants. Am J Clin Nutr 2014;99(3):734S-41S.

Khymenets O, Rabassa M, Rodriguez-Palmero M, Rivero-Urgell M, Urpi-Sarda M, Tulipani S, et al. Dietary epicatechin is available to breastfed infants through breast milk in the form of host and microbial metabolites. J Agric Food Chem 2016;64(26):5354-5360. Available from: http:// pubs.acs.org/doi/10.1021/acs.jafc.6b01947 doi: 10.1021/acs.jafc.6b01947.

Koletzko B, Thiel I, Abiodun PO. The fatty acid composition of human milk in Europe and Africa. J Pediatr 1992;120:S62-70.

Koletzko B, Rodriguez-Palmero M. Polyunsaturated fatty acids in human milk and their role in early infant development. J Mammary Gland Biol Neoplasia 1999;4(3):269-84. 
Korman A, Oh A, Raskind A, Banks D. Statistical methods in metabolomics. In: Anisimova $\mathrm{M}$, editor. Evolutionary Genomics: Statistical and Computational Methods. 2012:381-413.

Landete JM. Updated knowledge about polyphenols: functions, bioavailability, metabolism, and health. Crit Rev Food Sci Nutr 2012;52(10):936-948. Available from: http://www.tandfonline.com/ doi/full/10.1080/10408398.2010.513779 doi: 10.1080/10408398.2010.513779.

LoCasio R, Ninonuevo M, Freeman S. Glycoprofiling of bifidobacterial consumption of human milk oligosaccharides demonstrates strain specific, preferential consumption of small chain glycans secreted in early human lactation. J Agric Food Chem 2007;55(22):2007-8914. Available from: http://pubs.acs.org/doi/abs/10.1021/ jf0710480 doi: 10.1021/jf0710480.

Longini M, Tataranno ML, Proietti F, Tortoriello M, Belvisi E, Vivi A, et al. A metabolomic study of preterm and term human and formula milk by proton MRS analysis: preliminary results. J Matern-Fetal Neonatal Med 2014;27(2):27-33. Available from: http://www.tandfonline.com/ doi/full/10.3109/14767058.2014.955958 doi: 10.3109/14767058.2014.955958.

Marincola FC, Dessì A, Corbu S, Reali A, Fanos V. Clinical impact of human breast milk metabolomics. Clin Chim Acta 2015;451:1036. Available from: http://linkinghub.elsevier.com/ retrieve/pii/S0009898115000856 doi: 10.1016/j. cca.2015.02.021.

Marincola FC, Noto A, Caboni P, Reali A, Barberini L, Lussu M, et al. A metabolomic study of preterm human and formula milk by high resolution NMR and GC/MS analysis: preliminary results. J Matern-Fetal Neonatal Med 2012;25(55):6267. Available from: http://www.tandfonline.com/ doi/full/10.3109/14767058.2012.715436 doi: 10.3109/14767058.2012.715436.

Newburg D, Ruiz-Palacios G, Morrow A. Human milk glycans protect infants against enteric pathogens. Annu Rev Nutr 2005;37-58.

Novak EM, Innis SM. Impact of maternal dietary n-3 and $n-6$ fatty acids on milk medium-chain fatty acids and the implications for neonatal liver metabolism. Am J Physiol Endocrinol Metab;2011(301):807-17. doi: 10.1152/ajpendo.00225.2011.

Praticò G, Capuani G, Tomassini A, Baldassarre ME, Delfini M, Miccheli A. Exploring human breast milk composition by NMR-based metabolomics. Nat Prod Res 2014;28(2):95-101. Available from: http://www.tandfonline.com/ doi/abs/10.1080/14786419.2013.843180 doi: 10.1080/14786419.2013.843180.

Qian L, Zhao A, Zhang Y, Chen T, Zeisel S, Jia W, et al. Metabolomic approaches to explore chemical diversity of human breast-milk, formula milk and bovine milk. Int J Mol Sci 2016;17(12):2128. Available from: http://www.mdpi.com/14220067/17/12/2128 doi: 10.3390/ijms17122128.

Ruiz-Palacios G, Cervantes L, Ramos P, ChavezMunguia B, Newburg D. Campylobacter jejuni binds intestinal $\mathrm{H}(\mathrm{O})$ antigen (Fuc alpha 1, 2Gal beta $1,4 \mathrm{GlcNAc}$ ), and fucosyloligosaccharides of human milk inhibit its binding and infection. J Biol Chem;278(16):2003-14112. Available from: http://www.jbc.org/lookup/doi/10.1074/jbc. M207744200 doi: 10.1074/jbc.M207744200.

Ryan EP, Heuberger AL, Broeckling CD, Borresen EC. Advances in Nutritional Metabolomics. Curr Metabolomics 2013;1(2):109-20. Available from: http://www.eurekaselect.com/ openurl/content. php? gen re $=$ article $\&$ issn $=2213$ 235X\&volume $=1$ \&issue $=2 \&$ spage $=109$ doi: $10.2174 / 2213235 X 11301020001$.

Sanders TAB, Reddy S. The influence of a vegetarian diet on the fatty acid composition of human milk and the essential fatty acid status of the infant. J Pediatr 1992;120:S71-7.

Savorani F, Rasmussen MA, Mikkelsen MS, Engelsen SB. A primer to nutritional metabolomics by NMR spectroscopy and chemometrics. Food Research International 2013;54(1):1131-1145. Available from: http://linkinghub.elsevier.com/ retrieve/pii/S0963996912005480 doi: 10.1016/j. foodres.2012.12.025.

Sharon N. Carbohydrate-lectin interactions in infectious disease. Adv Exp Med Biol 1996;1-8.

Shaw GM, Carmichael SL, Yang W, Selvin S, Schaffer DM. Periconceptional dietary intake of choline and betaine and neural tube defects in offspring. Am J Epidemiol 2004;160(2):102-9. Available from: https://academic.oup.com/aje/article-lookup/ doi/10.1093/aje/kwh187 doi: 10.1093/aje/kwh187.

Shoji H, Taka H, Kaga N, Ikeda N, Kitamura T, Miura Y, et al. A pilot study of the effect of human breast milk on urinary metabolome analysis in infants. J Pediatr Endochrinol Metab;30(9):2017-939. Available from: http://www.degruyter.com/view $/ \mathrm{j} /$ 
jpem.2017.30.issue-9/jpem-2017-0179/jpem2017-0179.xml doi: 10.1515/jpem-2017-0179.

Smilowitz J, Sullivan, A., O'Barile, D. , German J, Lonnerdal B, Slupsky C. The human milk metabolome reveals diverse oligosaccharide profiles. J Nutr;143(11):2013-1709. Available from: http:// jn.nutrition.org/cgi/doi/10.3945/jn.113.178772 doi: $10.3945 /$ jn.113.178772.

Su SV, Gurney KB, Lee B. Sugar and spice: viral envelope-DCSIGN interactions in HIV pathogenesis. Current HIV Res 2003;1(1):8799. Available from: http://www.eurekaselect.com/ openurl/content. php? genre $=$ article $\& i s s n=1570$ $162 \mathrm{X} \&$ volume $=1$ \&issue $=1 \&$ spage $=87$ doi: $10.2174 / 1570162033352129$.

Sumayao R, Madriaga JR, Perlas LA, Cheong RL, Desnacido JA, Marcos JM, et al. Breastmilk retinol of Filipino lactating women and the vitamin A status of their breast-fed infants. Phil J Nutr;55:18-26.

Sundekilde U, Downey E, O'Mahony J, O'Shea CA, Ryan C, Kelly A, et al. The Effect of Gestational and Lactational Age on the Human Milk Metabolome. Nutrients 2016;8(5):304. Available from: http:// www.mdpi.com/2072-6643/8/5/304 doi: 10.3390/ nu8050304.

Sundekilde UK, Larsen LB, Bertram HC. NMR-based milk metabolomics. Metabolites 2013;3(2):204-22. Available from: http://www. mdpi.com/2218-1989/3/2/204 doi: 10.3390/ metabo3020204.

Totten SM, Zivkovic AM, Wu S, Ngyuen UT, Freeman SL. Ruhaak LR, Darboe MK, German JB, Prentice AM, Lebrilla CB. Comprehensive profiles of human milk oligosaccharides yield highly sensitive and specific markers for determining secretor status in lactating mothers. J Proteome Res 2012;2012(11):6124-6133. Available from: http:// pubs.acs.org/doi/abs/10.1021/pr300769g doi: $10.1021 / \mathrm{pr} 300769 \mathrm{~g}$.

Liempt, E. Van , Bank CM, Mehta P, et al. Specificity of DC-SIGN for mannose- and fucosecontaining glycans. FEBS Lett 2006;580(26):61236131. Available from: http://doi.wiley. com/10.1016/j.febslet.2006.10.009 doi: 10.1016/j. febslet.2006.10.009.

Villaseñor A, Garcia-Perez I, Garcia A, Posma JM, Fernández-López $\mathrm{M}$, Nicholas $\mathrm{AJ}$, et al. Breast milk metabolome characterization in a single-phase extraction, multiplatform analytical approach. Anal Chem 2014;86(16):8245-52. Available from: http://pubs.acs.org/doi/10.1021/ac501853d doi: $10.1021 /$ ac $501853 \mathrm{~d}$.

Wishart DS, Tzur D, Knox C, Eisner R, Guo AC, Young N, et al. HMDB: the human metabolome database. Nucleic Acids Res 2007;35(1). doi: 10.1093/nar/gkl923.

Wishart DS. Applications of metabolomics in drug discovery and development. Drugs R D 2008;9(5):307-22. Available from: http://link. springer.com/10.2165/00126839-200809050 00002 doi: 10.2165/00126839-200809050-00002.

World Health Organization, (UNICEF) . . Global strategy for infant and young child feeding: World Health Organization; 2003.

Wu J, Domellöf M, Zivkovic AM, Larsson G, Öhman A, Nording ML. NMR-based metabolite profiling of human milk: A pilot study of methods for investigating compositional changes during lactation. Biochemical and Biophysical Research Communications 2016;469(3):626-632. Available from: http://linkinghub.elsevier.com/ retrieve/pii/S0006291X15309839 doi: 10.1016/j. bbrc.2015.11.114.

Xia J, Wishart DS. Web-based inference of biological patterns, functions and pathways from metabolomic data using MetaboAnalyst. Nat Protoc 2011;6(6):743-6. Available from: http://www. nature.com/doifinder/10.1038/nprot.2011.319 doi: 10.1038/nprot.2011.319.

XiangM,AlfvenG, Blennow M, Trygg M,Zetterström R. Long-chain polyunsaturated fatty acids in human milk and brain growth during early infancy. Acta Paediatr 2000;89(2):142-7. Available from: http://doi.wiley.com/10.1111/j.1651-2227.2000. tb01206.x doi: 10.1111/j.1651-2227.2000. tb01206.x.

Zeisel SH, Blusztajn JK. Choline and Human Nutrition. Annu. Rev. Nutr 1994;14(1):269-296. Available from: http://www.annualreviews.org/ doi/10.1146/annurev.nu.14.070194.001413 doi: 10.1146/annurev.nu.14.070194.001413. 